\title{
LIFTING SETS AND THE CALKIN ALGEBRA
}

\author{
by G. J. MURPHY
}

(Received 22 August, 1980)

$H$ will denote a Hilbert space of infinite dimension, $\mathscr{B}(H)$ the algebra of bounded linear operators on $H$, and $\mathscr{H}(H)$ the ideal of compact operators on $H$. We let $\sigma, \sigma_{e}$ and $\sigma_{\omega}$ denote the spectrum, essential spectrum and Weyl spectrum respectively. It is well known that for arbitrary $T \in \mathscr{B}(H)$ we have by [5]

and

$$
\sigma_{e}(T)=\sigma(T+\mathscr{K}(H))=\sigma(T) \backslash\{\lambda \in \sigma(T): \lambda 1-T \text { is a Fredholm operator }\}
$$

$$
\begin{aligned}
\sigma_{\omega}(T) & =\bigcap_{K \in \mathscr{H}(H)} \sigma(T+K) \\
& =\sigma(T) \backslash\{\lambda \in \sigma(T): \lambda 1-T \text { is a Fredholm operator of index zero }\}
\end{aligned}
$$

and

$$
\sigma_{e}(T) \subseteq \sigma_{\omega}(T) \subseteq \sigma(T)
$$

$\Delta$ will always denote a non-empty compact subset of the plane. We say $\Delta$ is a lifting set (for $H$ ) if for every $T \in \mathscr{B}(H)$ with $\sigma_{e}(T)=\Delta$ there exists $K \in \mathscr{K}(H)$ such that $\sigma(T+K)=\Delta$. (It is easily seen that there is always some $T \in \mathscr{B}(H)$ with $\sigma_{e}(T)=\Delta$.) We show that $\Delta$ is a lifting set if and only if it has no holes. The following theorem is stated for reference (see also [1]). The result is due to Stampfli [6].

Theorem 1. For any $T \in \mathscr{B}(H)$ there exists $K \in \mathscr{K}(H)$ such that $\sigma(T+K)=\sigma_{\omega}(T)$.

The following result is immediate from this theorem.

Lemma 2. $\Delta$ is a lifting set if and only if $\sigma_{e}(T)=\sigma_{\omega}(T)$ for every $T \in \mathscr{B}(H)$ such that $\sigma_{e}(T)=\Delta$.

THEOREM 3. $\Delta$ is a lifting set if and only if $\Delta$ is polynomially convex.

Proof. Suppose $\Delta$ is a lifting set, but is not polynomially convex. Let $\omega$ be any hole of $\Delta$. It is a consequence of Berger and Shaw [2] that there exists $T \in \mathscr{B}(H)$ with $\sigma_{e}(T)=\Delta$ and ind $(\lambda I-T)=1(\lambda \in \omega)$. (Here ind denotes the Fredholm index.) Now since $\Delta$ is a lifting set, $\sigma_{\omega}(T)=\sigma_{e}(T)$ by the Lemma. But

$$
\sigma_{\omega}(T)=\sigma_{e}(T) \cup\{\lambda: \lambda I-T \text { is Fredholm and ind }(\lambda I-T) \neq 0\} .
$$

Hence $\omega \subseteq \sigma_{\omega}(T)=\sigma_{e}(T)=\Delta$. This contradiction shows that lifting sets have no holes.

Conversely, suppose $\Delta$ is polynomially convex, and let $\sigma_{e}(T)=\Delta$. By Stampfli's theorem there exists $K \in \mathscr{K}(H)$ with $\sigma_{\omega}(T)=\sigma(T+K)$. Also, $\sigma_{\omega}(T)$ consists of $\sigma_{e}(T)$ with some of its holes [4], and so in this case $\Delta=\sigma_{e}(T)=\sigma_{\omega}(T)=\sigma(T+K)$. Thus $\Delta$ is a lifting set.

It is interesting to note that the well-known result of West [7] that a Riesz operator $T$ can be written $T=Q+K$, where $Q$ is quasinilpotent and $K \in \mathscr{K}(H)$, says precisely that $\{0\}$

Glasgow Math. J. 23 (1982) 83-84. 
is a lifting set. (By the Ruston characterization of Riesz operators [3 p. 43], $T$ is Riesz if and only if $\sigma_{e}(T)=\{0\}$.) It is unknown whether the West decomposition of a Riesz operator holds in arbitrary Banach spaces.

\section{REFERENCES}

1. C. Apostol, C. Pearcy and N. Salinas, Spectra of compact perturbations of operators, Indiana Univ. Math. J. 26 (1977), 345-350.

2. C. A. Berger and B. I. Shaw, Self-commutators of multi-cyclic hyponormal operators are always trace-class, Bull. Amer. Math. Soc. 79 (1973), 1193-1199.

3. S. R. Caradus, W. E. Pfaffenberger, B. Yood, Calkin algebras and algebras of operators on Banach spaces, (Marcel Dekker, New York, 1974).

4. P. A. Fillmore, J. G. Stampfli and J. P. Williams, On the essential numerical range, the essential spectrum, and a problem of Halmos, Acta Sci. Math. (Szeged) 33 (1972), 179-192.

5. M. Schechter. Invariance of the essential spectrum, Bull. Amer. Math. Soc., 71 (1965), 365-367.

6. J. G. Stampfli, Compact perturbations, normal eigenvalues and a problem of Salinas. $J$. London Math. Soc. (2), 9 (1974), 165-175.

7. T. T. West. The decomposition of Riesz operators. Proc. London Math. Soc. (3), 16 (1966), 737-752.

School of Mathematics

Trintty College,

DUBLIN 